\title{
A randomized controlled trial to study the effect of a pediatric anesthesia comic information leaflet on preoperative anxiety in children
}

\author{
Malavika M. Kulkarni ${ }^{1}$, Shwetha Pradeep ${ }^{2} \bowtie$, Madhu Rao ${ }^{3} \bowtie$, Muralidhar M. Kulkarni ${ }^{4} \bowtie$ \\ Author affiliation: \\ 1. Department of Anesthesia, Kasturba Medical College, Manipal Academy of Higher Education, Manipal, India. \\ 2. Mysore Medical College \& Research Institute. Mysuru, Karnataka 570001, India \\ 3. Consultant Anesthesiologist, NMC Royal Hospital, Khalifa City, Abu Dhabi, UAE. \\ 4. Department of Community Medicine, Kasturba Medical College, Manipal Academy of Higher Education, Manipal, India.
}

Correspondence: Dr. Malavika Kulkarni; E-mail: malavika.kulkarni@manipal.edu; Phone: +91-9844241620

\section{Abstract}

Background \& Objective: Preoperative anxiety among children may at times be very cumbersome for the anesthesiologist, as an anxious child cannot be expected to be very cooperative. Anxiety may interfere into vascular excess and smooth induction of anesthesia. This study was conducted to assess the efficacy of 'pediatric anesthesia comic information' leaflet in reducing preoperative anxiety and to obtain parents feedback on its utility.

Methodology: It was a prospective single blinded randomized controlled trial among 150 children, aged 6-12 y, undergoing elective surgery in a tertiary-care hospital. Test group (Group T) was given a comic leaflet along with oral information while control group (Group C) received only oral information. Preoperative anxiety was measured using Modified Yale Preoperative Anxiety Scale (m-YPAS) at three time intervals along with feedback from parents on the comic's utility. Data was analyzed using SPSS version 21.0 with Chi-square, independent t and Mann Whitney tests applied to interpret the results.

Results: Pre-operative anxiety on the day prior to surgery (T1) was $25.75 \pm 5.52$ and $26.61 \pm 7.77$ compared to baseline (TO) score of $25.8 \pm 6.1$ and $26.6 \pm 8.0$ in group T and C respectively. The mean scores in the holding area (T2) was $38.87 \pm 14.20$ and $41.35 \pm 16.40$ in Group $T$ and $C$ respectively. The $p$ value of change in the preoperative anxiety scores T0-T1 was 0.2 , and T0-T2 was 0.24 with no significant decrease in preoperative anxiety in intervention compared to the control group ( $p>0.05)$.

Conclusion: Our results show that comic leaflet, given pre-operatively in addition to standard oral information had no additional effect on reducing anxiety in children undergoing surgery. However, the parents of the study subjects found the comic leaflet to be informative and it needs to be explored further in future research.

Key words: Anesthesia; Preoperative anxiety; Pediatrics; Surgery; Comic leaflet

Citation: Kulkarni MM, Pradeep S, Rao M, Kulkarni MM. A randomized controlled trial to study the effect of a pediatric anesthesia comic information leaflet on preoperative anxiety in children. Anaesth. pain intensive care 2021;26(1):75-80. DOI: 10.35975/apic.v26i1.1771

Received: June 27, 2021, Reviewed: September 28, 2021, Accepted: November 17, 2021

\section{Introduction}

Preoperative anxiety is reported by nearly $60 \%$ of all young children undergoing surgery, ${ }^{1}$ with several negative outcomes that include lack of cooperation during venous cannulation, refusing to be separated from the parents, refusing to lie on operating table, and mask refusal etc. It may also lead to increased anesthetic requirements, increased postoperative pain, emergence delirium, sleep disturbances and other maladaptive behavioral changes, which can last for weeks following surgery. ${ }^{2,3}$ Hence it is important that we address 
preoperative anxiety in order to avoid the probable secondary effects.

Several methods have been developed to combat preoperative anxiety, comprising pharmacological as well as non-pharmacological measures. The pharmacological methods include use of medications like midazolam, ketamine, clonidine etc. ${ }^{4}$ While, nonpharmacological ones are preparation programs that include distraction techniques, such as play therapy, comic cartoons and video games, which aim at educating the child and parent. ${ }^{5-8}$ Use of printed materials in reducing anxiety is a relatively unexplored subject in India. Comic information leaflet is a creative idea to convey something serious to children in a medium they would understand and enjoy. These are simple, cheap and effective method of reducing anxiety in children. ${ }^{9}$ Hence, the present study was aimed to determine the effect of a comic leaflet on preoperative anxiety among children and to compare requirement of preoperative anxiolytics on the day of surgery and also to assess the utility of the comic leaflet from the parents point of view.

\section{Methodology}

This prospective, single-blinded randomized control study was carried out involving children 6-12 y of age, ASA physical status I and II, who were enrolled in school class equivalent for his or her age (not held back by any year) posted for elective surgery under general anesthesia. Children with psychomotor deficits, those on psychoactive drugs, any development delay, hearing and visual impairment, posted for major surgeries and those with both parent's being illiterate were excluded. Children who did not read the comic leaflet or who had not been sent to school and children who had undergone surgery previously were also excluded. Written informed consent from parent/guardian along with an assent from children who were older than seven years was taken prior to the study. Patients were randomly assigned to the control group (Group C) or the intervention group (Group T) using a computer-generated randomization table. Concealment was ensured using sequentially numbered, opaque sealed envelopes.

Our primary objective was to assess the change in preoperative anxiety with pediatric anesthesia comic information leaflet amongst children. The preoperative anxiety of the child was assessed with Modified Yale Preoperative Anxiety Scale (m-YPAS) before providing the comic information leaflet at admission (T0) and a second assessment (T1) was done at the end of the day by the same observer and this process was not blinded. The m-YAPS is an observational state anxiety measure for young children consisting of 27 items under five activity domains that include emotional expressivity, vocalization, state of arousal and use of parents. The scores range from 22.9 to 100 and a cut off value of 30 was used to classify into anxious and non-anxious.

All the patients were evaluated one day prior to the surgery in the in-patient ward by observer- 1 who was a postgraduate resident in anesthesiology. Children belonging to Group $\mathrm{C}$ and their parents received verbal information, as routinely provided by the anesthetist during the pre-anesthetic consultation. They received information on fasting, hospitalization, anesthetic procedures, risk of anesthesia, pain management during and after surgery. Children belonging to Group $\mathrm{T}$ and their parents/guardians received a comic information leaflet titled "Raju's amazing journey to sleepy land" explaining each step of the anesthetic procedure from the point of admission to the hospital till discharge. It contained information on IV cannulation, pre anesthetic evaluation, fasting, and transport to operation theatre, induction and recovery. Each step was accompanied by a short explanation and colorful illustration. The text was in simple language which could be easily understood by children. The quality and usefulness of the leaflet was initially evaluated by the departmental scientific review committee comprising of a panel of experts in pediatric anesthesia. A pilot study was then conducted amongst 10 children speaking three different languages and refined further based on their feedback. The parent/guardian was allowed to participate in the discussion with observer-1. In addition, they also received the routine oral information by the same observer-1 as it is the standard care provided in the hospital. The comic was originally developed in English and translated to Kannada, Malayalam and other languages by language experts followed by back translation to ensure adherence to the original version and was distributed as per the language preference of the child.

The image of the comic leaflet with few illustrations is shown in Figure 1.

The level of education and language spoken by the parent/guardian was taken into account to decide the language of the comic leaflet to be provided and to ensure that parents can read it to assist their children to comprehend the details in the leaflet and give feedback about its utility. The comic leaflet was provided on the morning of admission and took 20 to $30 \mathrm{~min}$ to read and the participants were given an opportunity to clarify their doubts or discuss with observer-1. The m-YPAS score (T1) was reassessed in the ward by observer-1 by the end of the day. Feedback on utility of the comic leaflet was collected from the parent/guardian at the same time.

On the day of surgery, the child was shifted to the dedicated pre-medication area of the operating room complex with the parents and a final m-YPAS (T2) was assessed by observer-2, a consultant anesthesiologist in the study and blinded to the group, at least half an hour 

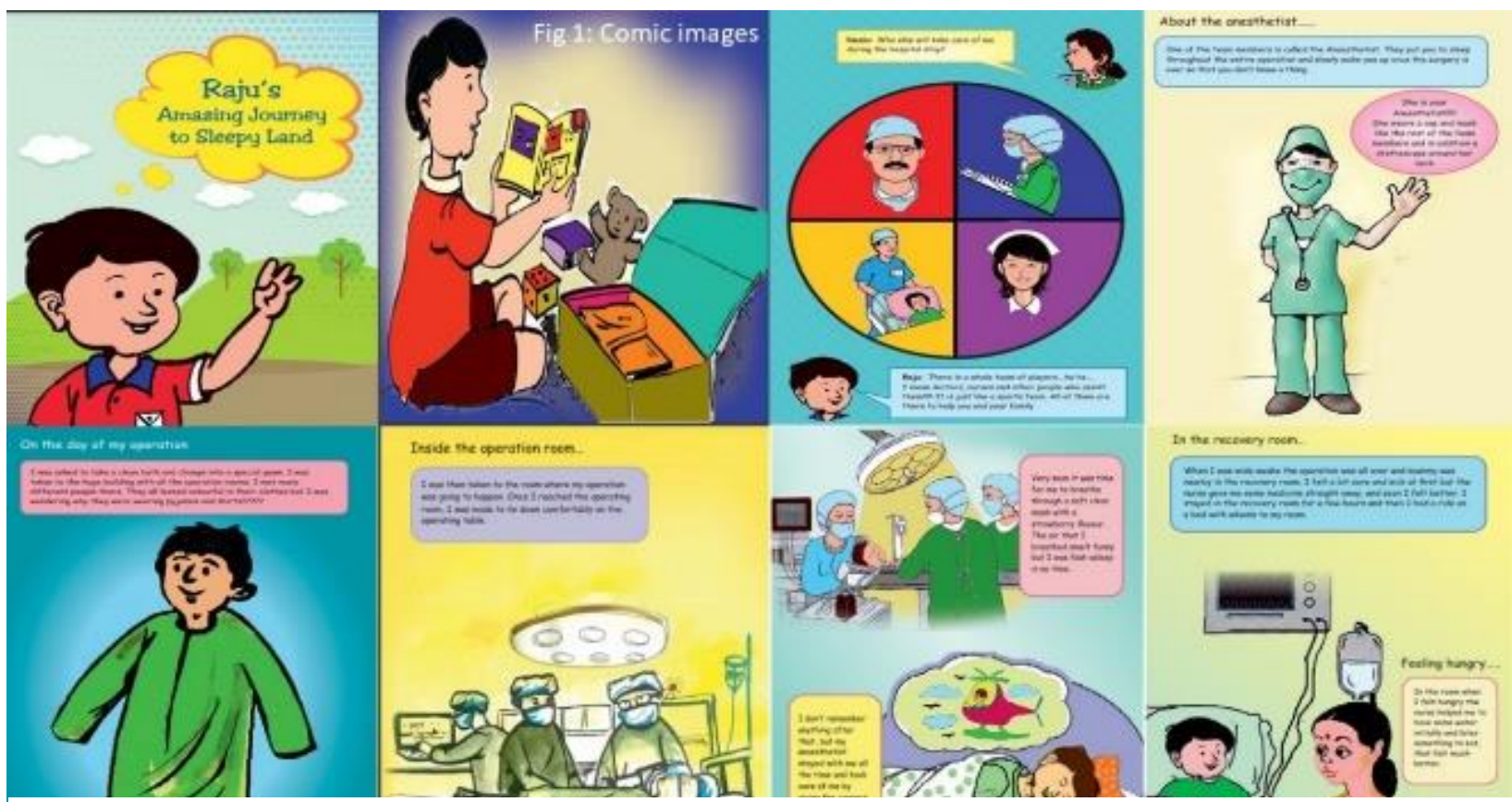

Figure 1: Images of Pediatric Anesthesia Comic Information Leaflet

prior to the surgery. Fasting status was confirmed and duration of fasting noted in all children. Just before shifting to the operating room, the parent separation anxiety was assessed by observer-2 using Parent Separation Anxiety Scale. ${ }^{11-12}$ If the score was $\geq 2$, anxiolysis was advised with either midazolam, 0.05 $\mathrm{mg} / \mathrm{kg}$ IV (if intravenous cannula was already secured) or oral midazolam $0.5 \mathrm{mg} / \mathrm{kg}$ in paracetamol syrup 20 $\mathrm{mg} / \mathrm{kg}$ (if there was no cannula).

Sample size calculation: The study sample size was based on the child's preoperative anxiety ratings assessed by $\mathrm{m}$-YPAS on the day prior to surgery and on the day of surgery. As per the discretion of the anesthesiologist, difference in m-YPAS anxiety score of 15 , (either increase or decrease) was considered significant and considering a two-tailed $\alpha$ value of 0.05 and $80 \%$ power of study, 75 children were needed to be recruited in each arm leading to a total of 150 participants.

Data analysis: Statistical analysis was done using SPSS version 21 for Windows 8. Chi square, Mann Whitney and Independent sample $t$ test were applied as appropriate to interpret the results. $\mathrm{P}<0.05$ was considered as statistically significant.

\section{Results}

This randomized control study included 75 participants each in both the groups over a period of 18 months from March 2018 to August 2019 as shown in Figure 2. A total of 172 children were enrolled to get the required sample size of 150 while 22 were excluded as they did not meet the inclusion criteria.
The age, gender, mean height and weight of patients were comparable in both the groups and there was no statistically significant difference between both the groups as depicted in Table 1.

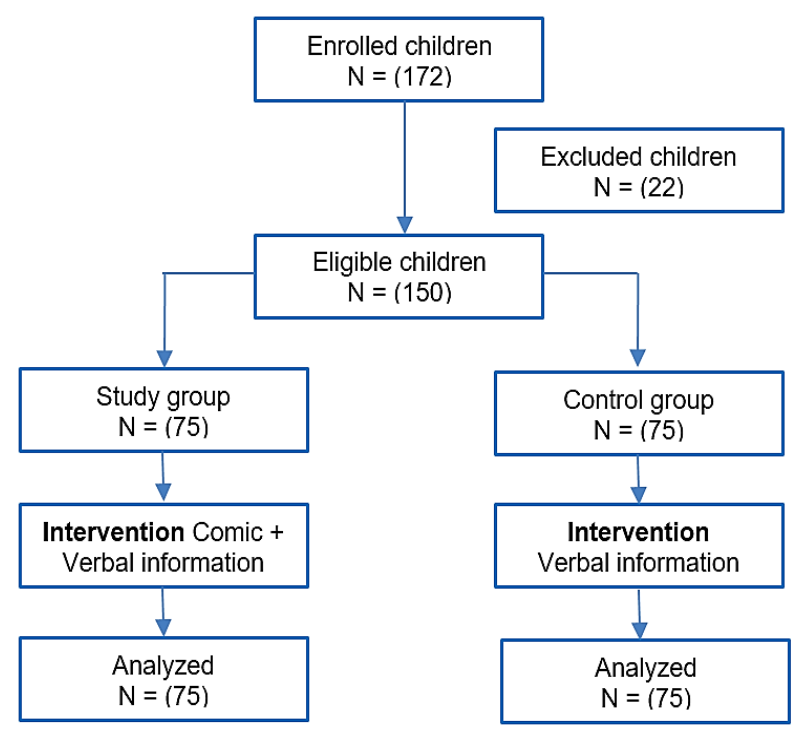

Figure 2: CONSORT flow diagram of the study

The type of surgery was similar in both groups and predominantly included herniotomy, orthopedic, otorhinolaryngology and urology surgeries with one case each belonging to dental extraction and percutaneous ASD device closure. None of the children had any other symptomatic comorbidity causing functional limitation. Most (96.0\%) of them were ASA I with few patients 
(4.0\%) graded as ASA II due to history of upper respiratory tract infection in the last three months. In terms of child's anxiety the mYPAS score at baseline (T0) was $25.8 \pm 6.1$ in group $\mathrm{T}$ and $26.8 \pm 8.0$ in group $\mathrm{C}$. The scores at $\mathrm{T} 1$ after providing comic leaflet or routine advice on the day prior to surgery were $25.8 \pm 5.5$ and $26.6 \pm 7.8$ in the test and control group respectively. The change in mean m-YPAS from baseline T0 to T1 did not reveal any significant decrease in anxiety on the day prior to surgery between the two groups $(\mathrm{P}=0.20)$. There was significant increase in the mean m-YPAS score on the day of surgery and observed to be $38.9 \pm 14.2$ in test group and $41.3 \pm 16.4$ in control group. However, the change in mean m-YPAS from T0 to T2 did not show any significant decrease in anxiety on the day of surgery $(\mathrm{P}=0.24)$. The score change of 15 or more considered as significant was not achieved and a pictorial description of the same is provided in CF 3 .

The total number of children requiring anxiolysis was more in control group $(73.3 \%)$ compared to the test group $(68.0 \%)$, and the difference was not statistically significant $(\mathrm{P}=0.45)$

Further, the utility of comic leaflet was elicited from parents and most of them $(97.3 \%)$ opined that the leaflet comforted them and they found it informative and easy to understand. Other details about their opinions are depicted in Table 2.

\section{Discussion}

This is the first randomized controlled trial, to the best of our knowledge, evaluating the effect of printed comic information leaflet on pre-operative anxiety among children undergoing elective surgeries carried out in South East Asian region,.
Table 1: Demographic and anthropometric details of study population

\begin{tabular}{|c|c|c|c|}
\hline Characteristics & Group C & Group T & ${ }^{*} p$ value \\
\hline${ }^{*}$ Age $(y)$ & $8.49 \pm 2.00$ & $8.48 \pm 2.17$ & 0.962 \\
\hline \#Gender (M/F) & $56 / 19$ & $48 / 27$ & 0.156 \\
\hline *Mean height(cm) & $124.77 \pm 10.39$ & $124.49 \pm 9.10$ & 0.867 \\
\hline${ }^{*}$ Mean weight $(\mathrm{kg})$ & $25.62 \pm 7.83$ & $25.4 \pm 9.103$ & 0.859 \\
\hline
\end{tabular}

In our study, we found that the pediatric anesthesia comic information leaflet coupled with standard verbal information had no additional effect in reducing preoperative anxiety in children as compared to standard verbal information provided before surgery. Further, children who received the intervention at pre-anesthetic visit did not have significant variation in the anxiety scores from the baseline on the day prior to surgery. However, on the day of surgery in the preoperative holding area, children in both the groups seemed to have heightened anxiety, thus neither benefit of comic information leaflet nor the standard verbal information seemed to exist.

The ideal manner of providing anesthesia information in the pre-operative period continues to be unresolved issue and hence various modes are being employed to reduce pre-operative anxiety in children. Several studies have been done in Western countries to evaluate the effect of preoperative preparation programs in alleviating preoperative anxiety in pediatric population. A similar randomized control trial done by Kassai et al. in France showed that a cartoon based anesthetic comic information leaflet was effective in reducing preoperative anxiety of children between six and seventeen years of age posted for elective surgery. Children who had received the leaflet up to one week before surgery, had a lower Speilberger State-Trait Anxiety Inventory (STAIC-S) scores on the day of hospitalization after pre-anesthetic visit which seemed to

\begin{tabular}{|c|c|c|}
\hline \multirow[t]{2}{*}{ Questions } & \multicolumn{2}{|c|}{ Parents response $\mathbf{N}(\%)$} \\
\hline & Yes & No \\
\hline Did you read the leaflet? $(\mathrm{N}=75)$ & $73(97.3)$ & $2(2.7)$ \\
\hline Did the leaflet comfort you? $(\mathrm{N}=73)$ & $71(97.2)$ & $2(2.8)$ \\
\hline Did the leaflet stress you? $(\mathrm{N}=70)$ & $3(4.3)$ & $67(95.7)$ \\
\hline Did you find the leaflet too complicated to understand? $(\mathrm{N}=70)$ & $2(2.9)$ & $98(97.1)$ \\
\hline Did you find the leaflet useful? $(\mathrm{N}=73)$ & $71(97.2)$ & $2(2.8)$ \\
\hline Did the leaflet give you information that you were unaware of? $(\mathrm{N}=73)$ & $71(97.2)$ & $2(2.8)$ \\
\hline $\begin{array}{l}\text { Did you feel that any information on experience of anesthesia and surgery } \\
\text { has not been addressed by the leaflet? }(\mathrm{N}=71)\end{array}$ & $0(0)$ & $100.0(71)$ \\
\hline
\end{tabular}


persist until the day of hospitalization $(\mathrm{p}=0.002)$ compared to the control group. They concluded that comic information leaflet was cheap and effective in lowering pre-operative anxiety. ${ }^{9,10}$

In another study, preoperative family centered ADVANCE multicomponent behavioral preparation program introduced 2-4 weeks before the surgical intervention was effective in reducing pre-operative anxiety with improvement in post-operative outcomes such as analgesic consumption, emergence delirium and time to discharge from post anesthesia care unit, compared with control group. ${ }^{13}$ It was probably the multi-pronged approach of interventions introduced several days prior to surgery that aided in achieving favorable outcomes compared to a single intervention in the present study. However, due to organizational limitations, lack of manpower and restricted financial resources, introducing comic information leaflet weeks prior to surgery was not feasible in our settings.

Different authors evaluated the efficacy of preoperative preparatory activities revealed that educational multimedia and web-based applications helped decrease pediatric preoperative anxiety, while the efficacy of therapeutic play and written materials remains uncertain. ${ }^{15}$ Studies have revealed that development of coping strategies is the most effective intervention preoperatively followed by role-play, therapeutic play and orientation tour of the OR with the child's age and the timing of intervention playing a key role. ${ }^{14,15}$

In future studies, it is advisable to intervene early and subsequently evaluate their responses on the day of surgery. While adopting a new preparatory program, besides efficacy, we also have to consider the preferences of children along with parental involvement and adequate time to process the information in addition to ageappropriate preparatory strategies in a variety of forms. ${ }^{15}$

Although we included children belonging to normal academic level for their age we did not objectively assess the child's or parent's IQ. So there was no concrete way to ascertain the level of understanding of the child about the comic leaflet. The leaflet could be tested among small subset of children fluent in three languages in the pilot study due to feasibility issues, however, it would be desirable to validate this leaflet among other stakeholders like parents and child psychologist too. Parental anxiety, which is a major contributor of pediatric anxiety was not assessed. ${ }^{16-17}$

There is a lack of awareness among general public about preoperative anxiety in children and its consequences. Efforts have to be made to educate parents of children coming for surgery about preoperative anxiety and the need for preparatory programs. Introduction of a comic information leaflet is just an initiative, it will take some time before such interventions gain acceptance and popularity. ${ }^{15}$ Many health centers abroad have designated pre-anesthesia clinics that have the resources to carry out these kinds of preoperative teaching programs which needs to be explored in India and other South East Asian countries.

\section{Limitations}

We did not objectively assess the child's or parent's IQ. So there was no concrete way to ascertain the level of understanding of the child about the comic leaflet. Parental anxiety, which is a major contributor of pediatric anxiety was not assessed. The first exposure of the child to the comic poster may need to be many days earlier than a day before, although it might not be easy considering logistic problems to the parents.

\section{Conclusion}

Pediatric comic information leaflet given on the day before surgery, although an educative strategy is insufficient to decrease preoperative anxiety among children in the intervention group. A positive note is that parents/guardian expressed that the comic leaflet was comforting, informative and easy to understand and could be explored further in future studies to tackle preoperative anxiety. In future studies, it may be necessary to provide sufficient time and reinforce written information leaflets with digital technology to allow the child to internalize the information provided.

\section{Conflict of interests}

None declared by the authors.

\section{Authors' contribution}

MMK: Concept, design of the study, developed the comic leaflet, supervising the data collection, data analysis and manuscript writing.

SP: Conduction of study, data entry, analysis, and manuscript writing

MR: Co-guide, concept design, supervising the data collection and editing the manuscript.

\section{References}

1. Fortier MA, Kain ZN. Treating perioperative anxiety and pain in children: a tailored and innovative approach. Paediatr Anaesth. 2015 Jan;25(1):27-35. [PubMed] DOI: 10.1111/pan.12546

2. Moura LA, Dias IM, Pereira LV. Prevalence and factors associated with preoperative anxiety in children aged 5-12 years. Rev Lat Am Enfermagem. 2016 Jun 14;24:e2708. [PubMed] DOI: 10.1590/1518-8345.0723.2708

3. Lee JH, Jung HK, Lee GG, Kim HY, Park SG, Woo SC. Effect of behavioral intervention using smartphone application for preoperative anxiety in pediatric patients. Korean J Anesthesiol. 

$2013 \quad$ Dec;65(6):508-18. [PubMed] DOI:
10.4097/kjae.2013.65.6.508

4. McCann ME, Kain ZN. The management of preoperative anxiety in children: an update. Anesth Analg. $2001 \mathrm{Jul} ; 93(1): 98-$ 105. [PubMed] DOI: $10.1097 / 00000539-200107000-00022$

5. Silva RD, Austregésilo SC, Ithamar L, Lima LS. Therapeutic play to prepare children for invasive procedures: a systematic review. J Pediatr (Rio J). 2017 Jan-Feb;93(1):6-16. [PubMed] DOI: 10.1016/j.jped.2016.06.005

6. Lee J, Lee J, Lim H, Son JS, Lee JR, Kim DC, Ko S. Cartoon distraction alleviates anxiety in children during induction of anesthesia. Anesth Analg. 2012 Nov; 115(5):1168-73. [PubMed] DOI: 10.1213/ANE.0b013e31824fb469

7. Kerimoglu B, Neuman A, Paul J, Stefanov DG, Twersky R. Anesthesia induction using video glasses as a distraction tool for the management of preoperative anxiety in children. Anesth Analg. 2013 Dec;117(6):1373-9. [PubMed] DOI: 10.1213/ANE.0b013e3182a8c18f

8. Cassady JF Jr, Wysocki TT, Miller KM, Cancel DD, Izenberg N. Use of a preanesthetic video for facilitation of parental education and anxiolysis before pediatric ambulatory surgery. Anesth Analg. 1999 Feb;88(2):246-50. [PubMed] DOI: 10.1097/00000539-199902000-00004

9. Kassai B, Rabilloud M, Dantony E, Grousson S, Revol O, Malik $\mathrm{S}$, et al. Introduction of a paediatric anaesthesia comic information leaflet reduced preoperative anxiety in children. $\mathrm{Br}$ J Anaesth. 2016 Jul;117(1):95-102. [PubMed] DOI: 10.1093/bja/aew154

10. Kain ZN, Mayes LC, Cicchetti DV, Bagnall AL, Finley JD, Hofstadter MB. The Yale Preoperative Anxiety Scale: how does it compare with a "gold standard"? Anesth Analg. 1997 Oct;85(4):783-8. [PubMed] DOI: 10.1097/00000539199710000-00012
11. Feld LH, Champeau MW, van Steennis CA, Scott JC. Preanesthetic medication in children: a comparison of oral transmucosal fentanyl citrate versus placebo. Anesthesiology. 1989 Sep;71(3):374-7. [PubMed]

12. Weldon BC, Watcha MF, White PF. Oral midazolam in children: effect of time and adjunctive therapy. Anesth Analg. 1992 Jul;75(1):51-5. [PubMed] DOI: 10.1213/00000539-19920700000010

13. Kain ZN, Caldwell-Andrews AA, Mayes LC, Weinberg ME, Wang SM, MacLaren JE, et al. Family-centered preparation for surgery improves perioperative outcomes in children: a randomized controlled trial. Anesthesiology. 2007 Jan;106(1):65-74. [PubMed] DOI: 10.1097/00000542200701000-00013

14. Baghele A, Dave N, Dias R, Shah H. Effect of preoperative education on anxiety in children undergoing day-care surgery. Indian J Anaesth. 2019 Jul;63(7):565-570. [PubMed] DOI: 10.4103/ija.IJA_37_19

15. Dai $Y$, Livesley J. A mixed-method systematic review of the effectiveness and acceptability of preoperative psychological preparation programmes to reduce paediatric preoperative anxiety in elective surgery. J Adv Nurs. 2018 May 13. [PubMed] DOI: $10.1111 /$ jan. 13713 .

16. Perry JN, Hooper VD, Masiongale J. Reduction of preoperative anxiety in pediatric surgery patients using age-appropriate teaching interventions. J Perianesth Nurs. 2012 Apr;27(2):6981. [PubMed] DOI: 10.1016/j.jopan.2012.01.003

17. Bellew M, Atkinson KR, Dixon G, Yates A. The introduction of a paediatric anaesthesia information leaflet: an audit of its impact on parental anxiety and satisfaction. Paediatr Anaesth. 2002 Feb;12(2):124-30. [PubMed] DOI: 10.1046/j.14609592.2002.00788.x 